\title{
ON GENERALIZED SOLUTIONS OF LOCALLY FUCHSIAN ORDINARY DIFFERENTIAL EQUATIONS
}

\section{G. I. MIRUMBE and J. M. MANGO}

Department of Mathematics

Makerere University

P.O. Box 7062

Kampala

Uganda

e-mail: mirumbe@cns.mak.ac.ug

mango@cns.mak.ac.ug

\begin{abstract}
We consider an $m$-th order constant coefficient locally Fuchsian ordinary differential equation at the origin

$$
\left(\nabla^{m}+r_{m-1}(0) \nabla^{m-1}+\ldots+r_{1}(0) \nabla+r_{0}(0)\right) y(x)=0,
$$

where $x \in \mathbb{R}, \nabla=x \frac{d}{d x}$ and prove that there exists generalized solutions to this equation with support on the positive halfline. A long the way, using our method, we establish similar conditions for existence of generalized solutions for a specialized ordinary differential equation proposed in [1].
\end{abstract}

2010 Mathematics Subject Classification: 34A37, 44A10, 34M05.

Keywords and phrases: locally Fuchsian, singular distributions, Dirac delta function, boundary values.

Received April 16, 2018

(C) 2018 Scientific Advances Publishers 


\section{Introduction}

A general ordinary differential equation on the real line with real polynomial coefficients can be expressed in the form

$$
a_{n}(x) y^{(n)}+a_{n-1}(x) y^{(n-1)}+a_{n-3}(x) y^{(n-3)}+\cdots+a_{1}(x) y^{\prime}+a_{0}(x) y=0,
$$

where $a_{i}(x), \forall i \in\{0,1, \cdots, n\}$ are functions of a single variable on the real line. The solutions to (1.1) depend on whether the leading polynomial $a_{n}(x)$ has zeros along the real line [3]. If the leading polynomial $a_{n}(x)$ has no zeros along the real line (the elliptic case), then there are a number of methods to solve such ODE's and the solutions consist of realanalytic functions (classical solutions) that is functions whose derivatives up to and including the highest order $n$ exist. Such solutions have been studied and could, for example, be found in [9], [3] or any other book on ordinary differential equations. Studies on the existence of polynomial solutions has been carried out by say [2] where the authors propose a new approach for investigating polynomial solutions based on elementary linear algebra. Laradji [14] considers a general $n$-th order linear ODE and provides the necessary and sufficient conditions for which it has a general polynomial solution. For the special case of Fuchsian ordinary differential equations the classical functions exist in general [3]. However, if the leading term has zeros on the real line (the non-elliptic case), then the ordinary differential equation (1.1) may have singularities and such classical solutions may not exist [19]. Generalized functions that is both distributional (singular support distributions) and weak functions exist as solutions in this particular case, for example, in [10], [11]. 
For the case of weak solutions, Wiener \& Shah [20], considered the ordinary differential equation;

$$
\sum_{i=0}^{n} a_{i}(t) x^{(n-i)}(t)=0
$$

with the coefficients $a_{i}(t) \in C^{M+n-i}$ in the neighbourhood of $t=0$, stated and proved specific conditions under which one would obtain nontrivial distribution solutions or the $m$-th order distribution solutions concentrated at $t=0$ assuming that the Equation (1.2) has a regular singularity at the origin. Kananthai [13] considered the ordinary differential equation of the form,

$$
P(y)=t y^{(n)}(t)+m y^{(n-1)}(t)+t y(t)=0,
$$

where $m$ is any integer and $n \geq 2$ for $t \in(-\infty, \infty)$ and proved by the use of the Laplace transform that the values of $m$ determine whether the solutions to (1.3) are classical or generalized solutions with halfline support on $\mathbb{R}$. Other results using the Laplace transform include [18] and [12]. Mandai [15] proves the existence of distribution solutions for every Fuchsian partial differential operator in the sense of Baouendi-Goulaouic with weight $m-k$. Amphon [1] using the Laplace transform proposed generalized solutions to a certain fourth order ODE,

$$
a_{4} x^{4} y^{(4)}+a_{3} x^{3} y^{\prime \prime \prime}(x)+a_{2} x^{2} y^{\prime \prime}(x)+a_{1} x y^{\prime}(x)+m y(x)=0,
$$

where $m \in \mathbb{Z}$ and $x \in \mathbb{R}$ that depend on the values of $m$ for the special case $a_{1}=a_{2}=a_{3}=a_{4}=1$. In this paper, we propose generalized solutions to locally Fuchsian ODE's for general values of the constants albeit using the theory on existence of distribution solutions with support on the positive halfline. This procedure involves the construction of distribitions whose well definedness is proved in [6], [4], and [5]. 


\section{Preliminaries}

\subsection{Locally Fuchsian differential equations}

Let $P$ denote the operator on the left hand side of (1.1). If the polynomial coefficient of the highest order derivative has some zeros on the real line with or without multiplicities then at each of the real zeros one can re-write (1.1) in the form that is herein reffered to as locally Fuchsian. A similar definition exists in [17].

Definition 1. The operator $P$ is locally Fuchsian at a real zero $x=a$, if it can be written in the form

$$
P=\nabla^{m}+r_{m-1}(x) \nabla^{m-1}+\ldots+r_{1}(x) \nabla+r_{0}(x),
$$

where $\left\{r_{\nu}(x)\right\} \forall v \in\{0,1,2, \ldots, m-1\}$ are germs of holomorphic functions at $x=a$ and $\nabla=(x-a) \frac{d}{d x}$.

Remark 1. $P$ in (2.1) is said to be locally Fuchsian on the real line if it is locally Fuchsian at every zero of the coefficient of the highest order derivative $a_{n}(x)$.

Let $P$ be a differential operator as defined in (2.1), locally Fuchsian at a zero $x=a$. We shall assume without loss of generality that $a=0$. Now, let

$$
\bar{P}=\nabla^{m}+r_{m-1}(0) \nabla^{m-1}+\ldots+r_{1}(0) \nabla+r_{0}(0)
$$

represent a constant coefficient locally Fuchsian differential operator at the origin.

By the Fundamental theorem of algebra, we can re-write (2.2) as

$$
\bar{P}=\prod\left(\nabla-\alpha_{\nu}\right)^{t_{\nu}}
$$

where $\left\{\alpha_{\nu}\right\}$ is a set of distinct complex numbers and each $t_{\nu}$ represents the multiplicity of a root and $t_{1}+t_{2}+\ldots+t_{k}=m$. 
Let $D$ be a small open disk of radius $\delta$ centered at the origin. Since every $r_{v}(0)$ is constant then it is holomorphic in $D$. In the simply connected set $D_{*}=D \backslash[0, \delta)$, for each pair $(\nu, j)$ with $0 \leq j \leq t_{\nu}-1$ there exists single valued branches of multivalued analytic functions of the form

$$
\rho_{\alpha_{\nu}, j}(x)=x^{\alpha_{\nu}} \cdot(\log x)^{j}
$$

These functions are the so-called Nilsson class functions and the logarithmic term comes in when there are multiplicities $\left(t_{v}>1\right)$ of the zero $\alpha_{\nu}$. With this notations we give a result on the general properties of the $\nabla$ operator, we give a well known classical result about this solutions that can be found in [9] and give here below a proof as it is restated for the purpose of our results in this paper.

Theorem 1. The solution set $\left\{f \in \mathcal{O}\left(D_{*}\right): P(f)=0\right\}$ with $P$ as in (2.2) is an m-dimensional vector space which has a basis consisting of functions of the form

$$
\rho_{\alpha_{\nu}, j}(x): 1 \leq \nu \leq k: 0 \leq j \leq t_{\nu-1} .
$$

Proof. We first show that the functions $\rho_{\alpha_{\nu}, j}(x)=x^{\alpha_{\nu}} \cdot(\log x)^{j}$ are solutions. The action of each $\left(\nabla-\alpha_{\nu}\right)$ on (2.3) gives,

$$
\left(\nabla-\alpha_{\nu}\right) \rho_{\alpha_{\nu}, j}(x)=j \rho_{\alpha_{\nu}, j-1}(x) .
$$

If $j=0$, then $x^{\alpha_{v}}$ is a solution. But if $j \neq 0$, then for each $t_{v}$ we have that,

$$
\left(\nabla-\alpha_{\nu}\right)^{t_{\nu}} \rho_{\alpha_{\nu}, j}(x)=j(j-1) \ldots\left(j-\left(t_{\nu}-1\right) \rho_{\alpha_{\nu}, j-t_{\nu}}(x)=0,\right.
$$

thus the functions $\rho_{\alpha_{\nu}, j}(x)$ are solutions to $P(f)=0$ since the operator $P$ is composed of only such products. What remains is to prove that the functions are linearly independent. This means that if $\sum_{i=1}^{m} c_{i} f_{i}=0$, then $c_{i}=0, \forall i$. We consider two general forms, 
(a) Suppose there are no logarithmic functions in the solutions $(j=0)$. This occurs when all $\alpha_{i}$ 's are distinct and the $m$ solutions are the individual solutions, $x^{\alpha_{1}}, x^{\alpha_{2}}, \ldots, x^{\alpha_{m}}$. Taking the action of $\nabla-\alpha_{1}$ on $\rho_{\alpha_{\nu}, j}(x)$, we have

$$
c_{2}\left(\alpha_{2}-\alpha_{1}\right) x^{\alpha_{2}}+c_{3}\left(\alpha_{3}-\alpha_{1}\right) x^{\alpha_{3}}+\ldots+c_{m}\left(\alpha_{m}-\alpha_{1}\right) x^{\alpha_{m}}=0
$$

(2.4) is holds true only if $c_{2}=c_{3}=\ldots=c_{m}=0$ since all the $\alpha_{i}$ 's are distinct. Choosing another factor of $P$ yields another set of coefficients being zero hence all the $c_{i}$ 's are zero.

(b) Assume without loss of generality that, at least one of the factors $\left(\alpha_{i}\right)$ is repeated. The solution set will be $\left\{\sum_{i=1}^{m-1} c_{i} x^{\alpha_{i}}+c_{m} x^{\alpha_{1}} \log x\right\}$. The action of $P$ with different factors as in (a) gives that all $c_{i}$ 's are zero thus the set of solutions is linearly independent. Thereby forming an $m$ dimensional basis for the solution space to $P$.

\subsection{Boundary value distributions $(\mathfrak{D b})$ on the real line}

We state in this subsection a necessary condition for the existence of a boundary value distribution attached to an analytic function $f(z)$ as one approaches the real axis locally from the two half planes, upper $H_{+}$ and lower $H_{-}$, respectively.

If $f(z)$ is an analytic function defined in the complex plane in a domain near the real axis such as the open rectangle defined by;

$$
\{(x, y): 0<x<a: 0<y<b\},
$$

where $a$ and $b$ are positive finite real numbers near the origin, then it is said to have moderate or temperate growth as one approaches the real axis if for each compact subinterval $\alpha \leq x \leq \beta$ of $(a, b)$ there exists some integer $N>0$ and a constant $C$ such that 


$$
|f(x+i y)| \leq C \cdot y^{-N}
$$

This moderate growth (2.5) is a necessary and sufficient condition for the complex valued function $f(z)$ to have a boundary value $f(x \pm i 0)$ given by a distribution $\mu(f)$ defined on the real axis by the limit

$$
f(x \pm i 0)=\lim _{\epsilon \rightarrow 0} \int_{0}^{a} g(x) f(x \pm i \epsilon) d x: g(x) \in C_{0}^{\infty}(0, a)
$$

Theorem 3.1.13 in [8].

Definition 1. The limit integral in (2.6) yields a linear functional on test functions which give a distribution denoted by $\mathfrak{b}(f)$, called the boundary value distribution of the analytic function $f(z)$.

\subsection{Distributions with compact support and their Cauchy transform}

Let $\mu \in \mathfrak{D} \mathfrak{b}$, be the space of boundary value distributions with compact support on the closed subset $[0,1]$ of the real $t$-line ( $\mu$ could be a singular distribution). If $z$ is fixed then the function

$$
t \mapsto \frac{1}{z-t},
$$

belongs to the class of $C^{\infty}(\mathbb{R})$ functions on the real $t$-line.

Definition 2. The Cauchy transform of $\mu$ denoted by $\mathcal{C}_{\mu}(z)$ is defined by the action of $\mu$ on a $C^{\infty}$ function $\frac{1}{z-t}$. This function is holomorphic everywhere in the complex $z$-plane except on $[0,1]$ and is defined by

$$
\mathcal{C}_{\mu}(z)=\mu<\frac{1}{z-t}>, \quad z \in \mathbb{C} \backslash[0,1]
$$


The Cauchy transform by definition is an analytic function in $\mathbb{C} \backslash[0,1]$. The Cauchy transform satisfies the moderate growth as we approach the real axis. If $f(x) \in C_{0}^{\infty}(\mathbb{R})$, then for every Cauchy transform of a boundary value distribution $\mu, \mathcal{C}_{\mu}(z)$, there exists two boundary value distributions, $\mathcal{C}_{\mu}(x+i 0)$ and $\mathcal{C}_{\mu}(x-i 0)$ defined by

$$
\mathcal{C}_{\mu}(x+i 0)<f>=\lim _{\epsilon \rightarrow 0} \int_{x \in \mathbb{R}} \mu<\frac{1}{x-t+i \epsilon}>f(x) d x
$$

and

$$
\mathcal{C}_{\mu}(x-i 0)<f>=\lim _{\epsilon \rightarrow 0} \int_{x \in \mathbb{R}} \mu<\frac{1}{x-t-i \epsilon}>f(x) d x
$$

Lemma 1. Given that $\mu \in \mathfrak{D} \mathfrak{b}$ with support on the interval $[0,1]$ including the case of singular support distributions, then the boundary value distributions of its Cauchy transform $\mathcal{C}_{\mu}(z)$ satisfies the equation

$$
\mu(f)=\frac{i}{2 \pi}\left\{\mathcal{C}_{\mu}(x+i 0)<f>-\mathcal{C}_{\mu}(x-i 0)<f>\right\}
$$

where $f(x) \in C_{0}^{\infty}(\mathbb{R})$.

Proof. See [16].

Remark 2. Lemma 1 shows that we can recover a distribution with compact support (singular support) via the boundary values of the Cauchy transform $\mathcal{C}_{\mu}(z)$. This also indicates that if a function of a complex variable that has moderate growth as one approaches the real line defines a boundary value distribution and extends as a distribution to the points of singularities with compact support at those points. The theory on boundary value distributions can also be reviewed in [8], [16], [7]. 


\subsection{The boundary value distributions of the analytic function} $f(z)=z^{\alpha}$, where $\alpha=\{-k, k\}, k \in \mathbb{N}$

The function $f(z)=z^{\alpha}$, where $z$ is a complex number $z=x+i y$, defined in $\mathbb{C} \backslash \mathbb{R}_{+}$as $e^{\alpha \log z}$, where $\log z$ is real for $z \in \mathbb{R}_{+}$has boundary values $(x+i 0)^{\alpha}$ as one approaches the real axis from the upper half plane and respectively $(x-i 0)^{\alpha}$ from the lower half plane. If $\mathfrak{R e}(\alpha)>0$, then the functions $(x \pm i 0)^{\alpha}$ are both entire in the complex $z$-plane. However, when $\alpha$ is a negative integer, $-k$ for $k \in \mathbb{N}$, then the boundary values are no longer entire functions as the function would satisfy the moderate growth condition of (2.5). We re-state below a lemma which could also be found in [16], [8].

Lemma 2. Let $k$ be a non-zero positive integer $(k \in \mathbb{N})$. The distribution $x^{-k}$ extends to the distribution $(-1)^{(k-1)} \cdot \frac{\delta_{0}^{(k-1)}}{(k-1)}$, where $\delta_{0}^{(k-1)}$ is the $(k-1)$-th derivative of the Dirac delta function at the origin.

Proof. We note that the function $z^{-k}$ for $k \in \mathbb{N}$ satisfies the moderate growth condition (2.5) and therefore has a boundary value distribution $(x+i 0)^{-k}$. Assume that $\phi(x) \in C_{0}^{\infty}(\mathbb{R})$. Let the distribution $<x_{+}^{\alpha}, \phi(x)>$ defined from the locally integrable function $x^{\alpha}$ be denoted by $I_{\alpha}(\phi(x))$ and given by

$$
I_{\alpha}(\phi(x))=\int_{0}^{\infty} x^{\alpha} \phi(x) d x
$$

The distribution in (2.9) is analytic for $\mathfrak{R e}(\alpha)>-1$ but in our case we assume $\alpha \rightarrow-k$. Applying the integration by parts formula once on the integral (2.9) gives 


$$
I_{\alpha}(\phi(x))=-\frac{1}{\alpha+1} \int_{0}^{\infty} x^{\alpha+1} \phi^{\prime}(x) d x=-\frac{I_{\alpha+1}\left(\phi^{\prime}(x)\right)}{\alpha+1} .
$$

Applying the integration by parts formula twice successively on the integral (2.9) yields

$$
I_{\alpha}(\phi(x))=\frac{1}{(\alpha+1)(\alpha+2)} I_{\alpha+2}\left(\phi^{\prime \prime}(x)\right) .
$$

Applying the same integration by parts formula $k$ times successively on the integral (2.9) gives

$$
I_{\alpha}(\phi(x))=\frac{(-1)^{k} I_{\alpha+k} \phi^{(k)}(x)}{(\alpha+1)(\alpha+2) \ldots(\alpha+k)} .
$$

Note that the right hand side of Equation (2.10) is analytic except for simple poles at $\alpha=-1,-2, \ldots,-k$.

At $\alpha=-k, k \in \mathbb{N}$, the residue of the function $I_{\alpha}(\phi(x))$ is computed by the formula

$$
\operatorname{res}\left(I_{\alpha}(\phi(x)),-k\right)=\lim _{\alpha \rightarrow-k}(\alpha+k) I_{\alpha}(\phi(x))
$$

Therefore

$$
\begin{aligned}
& \operatorname{res}\left(I_{\alpha}(\phi(x)),-k\right)=\lim _{\alpha \rightarrow-k}(\alpha+k) \cdot \frac{(-1)^{k} I_{\alpha+k} \phi^{(k)}(x)}{(\alpha+1)(\alpha+2) \ldots(\alpha+k)}, \\
& \operatorname{res}\left(I_{\alpha}(\phi(x)),-k\right)=\frac{(-1)^{k} I_{0}\left(\phi^{(k)}(x)\right)}{(-1)^{k}(k-1)(k-2) \ldots 1}=\frac{\left(\phi^{(k-1)}(0)\right)}{(k-1) !}
\end{aligned}
$$

Since $<\delta_{0}^{(k-1)}, \phi(x)>:=(-1)^{(k-1)} \cdot \frac{\phi^{(k-1)}(0)}{(k-1) !}$, then it means that

$$
(\alpha+k) x_{+}^{\alpha} \rightarrow(-1)^{(k-1)} \cdot \frac{\delta_{0}^{(k-1)}}{(k-1) !} \text { as } \alpha \rightarrow-k
$$


Therefore

$$
x_{+}^{\alpha}-(-1)^{(k-1)} \cdot \frac{\delta_{0}^{(k-1)}}{(\alpha+k)(k-1) !} \rightarrow x_{+}^{-k} \text { as } \alpha \rightarrow-k .
$$

The following theorem gives the relationship between rational function solutions and distributional solutions to an ODE.

Theorem 2. If an ordinary differential equation (1.1) admits a rational function solution, then it also has a distributional solution of order $m$.

Proof. See [16] and [19].

\section{Main Results}

Consider the locally Fuchsian differential equation (2.2) with the complex variable $z=x+i y$ for $x, y \in \mathbb{R}$.

Theorem 3. If $P$ is a locally Fuchsian ordinary differential operator on the real line with constant coefficients as in (2.2), then there exists a distribution u with support on the positive halfline that can be extended to a distribution $u_{0}$ in the neighbourhood of 0 such that $P\left(u_{0}\right)=0$ and $0 \in \operatorname{supp}\left(u_{0}\right) \subset$ positive halfline.

Proof. Let $D$ be a small open disk of radius $\delta>0$ centered at the origin such that every complex function $f(z)$ is holomorphic in $D$. By Theorem 1 , in the simply connected set $D_{*}=D \backslash[0, \delta)$, for each pair $(\nu, j)$ with $0 \leq j \leq t_{\nu}-1$, there exists single valued branches of multivalued analytic functions of the form

$$
\rho_{\alpha_{\nu}, j}(z)=z^{\alpha_{\nu}} \cdot(\log z)^{j}
$$

If $\mathbb{R e}(\alpha)>0$, then the defined distribution will be an entire function that does not satisfy moderate growth condition (2.5). However, if $\mathbb{R} e(\alpha)>-1$, then we consider the following two cases: 
(a) The case where there are distinct roots to the indicial equation that is the case when $j=0$ in (3.1). Define $x_{+}^{\alpha}$ by

$$
x_{+}^{\alpha}:= \begin{cases}x^{\alpha}, & \text { for } x>0 \\ 0, & \text { for } x \leq 0 .\end{cases}
$$

The function $x_{+}^{\alpha}$ is locally integrable and depends on the parameter $\alpha$ therefore is a distribution for $\mathbb{R} e(\alpha)>-1$. This distribution can be extended outside the set $S=\{-1,-2,-3, \ldots\}$ meromorphically on $\mathbb{C}$ since it is analytic elsewhere on $\mathbb{C}$ except at the simple poles, see [7]. The points of the set $S$ are considered as simple poles of the resulting distribution of the form obtained in Lemma 2,

$$
x_{+}^{\alpha}-(-1)^{(k-1)} \cdot \frac{\delta_{0}^{(k-1)}}{(\alpha+k)(k-1) !} \rightarrow x_{+}^{-k} \text { as } \alpha \rightarrow-k .
$$

The distribution (3.2) can be normalized by an appropriate Gamma function that has the similar singularities $\Gamma(\alpha+1)$ to have, $u_{*}=\frac{x_{+}^{-k}}{\Gamma(k+1)}$ that is entire on $\mathbb{C}$. This distribution $\left(u_{*}\right)$ has support on the positive halfline and by Lemma 2 the distribution $x_{+}^{-k}$ extends to the singular distribution $C \delta_{0}^{(k-1)}$ with support at the origin, where $C$ is a constant depending on $k$. By Theorems 1 and 2, the distribution $u_{*}$ and the extension $C \delta_{0}^{(k-1)}$ of $x_{+}^{-k}$ are solutions to $P(u)$, where $u=u_{*}+C \delta_{0}^{(k-1)}$ for an appropriate constant $C$.

(b) The case where there are repeated roots to the indicial equation that is $j \geq 1$ in (3.1). This implies that the solutions contain powers of the logarithmic term. In this case, we define a regularization of each of 
the locally integrable functions involved that is $z^{\alpha}$ and $(\log z)^{j}$ thereby defining the following analytic densities:

$$
x_{+}^{\alpha}:= \begin{cases}x^{\alpha}, & \text { for } x>0, \\ 0, & \text { for } x<0,\end{cases}
$$

and

$$
\left(\log x_{+}\right)^{j}:= \begin{cases}(\ln x)^{j}, & \text { for } x>0 \\ 0, & \text { for } x<0 .\end{cases}
$$

With the possible extension that both of these analytic densities are zero at $x=0$. The existence of distributions of this form and their compositions and products are proved in the series of papers [6], [4], and [5]. With derivatives taken in the distribution sense it yields that $P(u)=0$, where $u=x_{+}^{\alpha}(\ln x)^{j}$.

Example 3.1. Consider a Fuchsian operator at the origin $P$ of the third order defined by $P:=x^{3} \frac{d^{3}}{d x^{3}}+6 x^{2} \frac{d^{2}}{d x^{2}}+7 x \frac{d}{d x}+1$ and seek the distribution solution space with support on the positive halfline with possible extension to singular distributions with support at 0 . The solution space of the operator $P$ is as follows.

We note that,

$$
\nabla=x \frac{d}{d x}, \nabla^{2}=x^{2} \frac{d^{2}}{d x^{2}}+x \frac{d}{d x}, \text { and } \nabla^{3}=x^{3} \frac{d^{3}}{d x^{3}}+3 x^{2} \frac{d^{2}}{d x^{2}}+x \frac{d}{d x}
$$

Using this quantities for $\nabla$ and its powers in the operator $P$, we get the equation

$$
\left(\nabla^{3}+3 \nabla^{2}+3 \nabla+1\right) f=0 .
$$


The indicial equation for (3.3) is $r^{3}+3 r^{2}+3 r+1=0$ and has one solution $r=-1$ appearing three times. This then means the distribution solution space is given by

$x_{+}^{-1}:=\left\{\begin{array}{ll}x^{-1}, & \text { for } x>0, \\ 0, & \text { for } x<0,\end{array} \quad x^{-1}\left(\log x_{+}\right):= \begin{cases}x^{-1} \log x, & \text { for } x>0, \\ 0, & \text { for } x<0\end{cases}\right.$

and

$$
x^{-1}\left(\log x_{+}\right)^{2}:= \begin{cases}x^{-1}(\log x)^{2}, & \text { for } x>0 \\ 0, & \text { for } x<0\end{cases}
$$

(a) For $f=x_{+}^{-1}$ with the extension that it is a Dirac measure at 0 (a consequence of Lemma 2) and $\varphi(x) \in \mathbb{C}^{\infty}(\mathbb{R})$. The action of $\nabla$ and its powers on $f$ is given by

$$
\begin{gathered}
\nabla(f)(\varphi)=-\int_{0}^{\infty} x^{-1}(x \varphi(x))^{\prime} d x=-\int_{0}^{\infty} x^{-1} \varphi(x) d x \\
\nabla^{2}(f)(\varphi)=\int_{0}^{\infty} x^{-1}\left(\left(x^{2} \varphi(x)\right)^{\prime \prime}-(x \varphi(x))^{\prime}\right) d x=\int_{0}^{\infty} x^{-1} \varphi(x) d x,
\end{gathered}
$$

and

$$
\nabla^{3}(f)(\varphi)=\int_{0}^{\infty} x^{-1}\left(-\left(x^{3} \varphi(x)\right)^{\prime \prime \prime}+3\left(x^{2} \varphi(x)\right)^{\prime \prime}-(x \varphi(x))^{\prime}\right) d x=-\int_{0}^{\infty} x^{-1} \varphi(x) d x
$$

substituting this values of $\nabla(f)$ and its powers shows that $f=x_{+}^{-1}$ is a solution in the distribution sense to (3.3) with support in the positive halfline. The extension of $f=x_{+}^{-1}$ as $\alpha \rightarrow-1$ that is $\delta_{0}$ is also a solution by Remark 3 below. 
(b) For $f=x^{-1}\left(\log x_{+}\right)$with the extension that it is zero at 0 and $\varphi(x) \in \mathbb{C}^{\infty}(\mathbb{R})$. The action of $\nabla$ and its powers on $f$ is given by

$$
\begin{aligned}
& \nabla(f)(\varphi)=-\int_{0}^{\infty} x^{-1}(\log x)(x \varphi(x))^{\prime} d x=\int_{0}^{\infty} x^{-1} \varphi(x) d x-\int_{0}^{\infty} x^{-1}(\log x) \varphi(x) d x \\
& \nabla^{2}(f)(\varphi)=\int_{0}^{\infty} x^{-1}(\log x)\left(\left(x^{2} \varphi(x)\right)^{\prime \prime}-(x \varphi(x))^{\prime}\right) d x=\int_{0}^{\infty}\left(x^{-1}(\log x)-2 x^{-1}\right) \varphi(x) d x
\end{aligned}
$$

and

$$
\begin{aligned}
\nabla^{3}(f)(\varphi) & =\int_{0}^{\infty} x^{-1}(\log x)\left(-\left(x^{3} \varphi(x)\right)^{\prime \prime \prime}+3\left(x^{2} \varphi(x)\right)^{\prime \prime}-(x \varphi(x))^{\prime}\right) d x \\
& =\int_{0}^{\infty}\left(-x^{-1}(\log x)+3 x^{-1}\right) \varphi(x) d x,
\end{aligned}
$$

substituting this values of $\nabla(f)$ and its powers proves that $f$ solves (3.3) in the distribution sense.

(c) For $f=x_{+}^{-1}(\log x)^{2}$ with the extension that it is zero at 0 and $\varphi(x) \in \mathbb{C}^{\infty}(\mathbb{R})$. The action of $\nabla$ and its powers on $f$ is given by,

$$
\begin{aligned}
\nabla(f)(\varphi)=-\int_{0}^{\infty} x^{-1}(\log x)^{2}(x \varphi(x))^{\prime} d x= & 2 \int_{0}^{\infty} x^{-1}(\log x) \varphi(x) d x \\
& -\int_{0}^{\infty} x^{-1}(\log x)^{2} \varphi(x) d x
\end{aligned}
$$

with the second power of $\nabla$ as

$$
\begin{aligned}
\nabla^{2}(f)(\varphi) & =\int_{0}^{\infty} x^{-1}(\log x)\left(\left(x^{2} \varphi(x)\right)^{\prime \prime}-(x \varphi(x))^{\prime}\right) d x \\
& =2 \int_{0}^{\infty} x^{-1} \varphi(x) d x-4 \int_{0}^{\infty} x^{-1}(\log x) \varphi(x) d x+\int_{0}^{\infty} x^{-1}(\log x)^{2} \varphi(x) d x
\end{aligned}
$$


and

$$
\begin{aligned}
& \nabla^{3}(f)(\varphi)=\int_{0}^{\infty} x^{-1}(\log x)^{2}\left(-\left(x^{3} \varphi(x)\right)^{\prime \prime \prime}+3\left(x^{2} \varphi(x)\right)^{\prime \prime}-(x \varphi(x))^{\prime}\right) d x \\
& =-6 \int_{0}^{\infty} x^{-1} \varphi(x) d x+6 \int_{0}^{\infty} x^{-1}(\log x) \varphi(x) d x-\int_{0}^{\infty} x^{-1}(\log x)^{2} \varphi(x) d x
\end{aligned}
$$

substituting this values of $\nabla(f)$ and its powers in (3.3) shows that $f$ is a distribution solution.

Remark 3. (i) The equation

$$
a_{4} x^{4} y^{(4)}+a_{3} x^{3} y^{\prime \prime \prime}+a_{2} x^{2} y^{\prime \prime}+a_{1} x y^{\prime}+m y=0,
$$

where $m \in \mathbb{Z}$ and $x \in \mathbb{R}$ and $a_{4}, a_{3}, a_{2}, a_{1}$, are constants is a special case of Theorem 3 above and solutions can be obtained as in this example.

(ii) The Dirac measure $f=\delta_{0}$ is a solution to (3.3) by Theorem 2 or Lemma 2.11 in [1].

\subsection{The generalized solutions for a fourth order Euler ordinary differential equation}

Consider the fourth order Euler differential equation:

$$
a_{4} x^{4} y^{(4)}+a_{3} x^{3} y^{\prime \prime \prime}(x)+a_{2} x^{2} y^{\prime \prime}(x)+a_{1} x y^{\prime}(x)+m y(x)=0,
$$

where $m \in \mathbb{Z}$ and $x \in \mathbb{R}$. Below we state a similar theorem to Theorem 3.1 in [1] and prove it using our method.

Theorem 4. The solutions to (3.5) depend on the values of $m$ and is given by the following cases if $a_{4}=a_{3}=a_{2}=a_{1}=1$ :

(a) If $m=-k^{4}-5 k^{3}-9 k^{2}-4 k$ for $k \in \mathbb{N}$, then there exists the distributional solutions of (3.5), which are singular distribution of the Dirac delta function and its derivatives.

(b) If $m=-k^{4}+5 k^{3}-9 k^{2}+4 k$ for $k \in \mathbb{N}$, then there exists weak solutions. 
Proof. (a) According to Theorem 3 and Theorem 2, such distributions come as extensions of $x_{+}^{\alpha}$ when $\alpha \rightarrow-k$. Let $y=x^{-k}$ and the derivatives exist as it is a locally integrable function. Differentiating $y(x)$ and substituting into the special case of (3.5) where $a_{4}=a_{3}=a_{2}=a_{1}=1$ gives

$$
x^{-k}[k(k+1)(k+2)(k+3)-k(k+1)(k+2)+k(k+1)-k+m]=0 .
$$

Since $x^{-k} \neq 0$, then $m=-k^{4}-5 k^{3}-9 k^{2}-4 k$ for $k \in \mathbb{N}$ for a distribution solution to exist.

(b) According to Theorem 3 such distributions exist when $\operatorname{Re}(\alpha)>-1$ that is let $\alpha=k$. Let $y=x^{k}$ and all the derivatives exist. Differentiating $y(x)$ with respect to $x$ and substituting in (3.5) gives

$$
x^{k}(k(k-1)(k-2)(k-3)+k(k-1)(k-2)+k(k-1)+k+m)=0 .
$$

Since $x^{k} \neq 0$ for $m=-k^{4}+5 k^{3}-9 k^{2}+4 k$ for $k \in \mathbb{N}$, then there exists weak solutions of the form $x_{+}^{k}$ with support on the positive halfline.

\section{Conclusion}

In this paper, we proved the existence of generalized solutions with support on the positive halfline for a general constant coefficient locally Fuchsian differential equation (2.2) by constructing distributions with support on the positive halfline. We notice that (3.5) is a special case of (2.2). However, one can still use the Laplace transform method as in [1] to find conditions that $m$ should satisfy for existence of generalized solutions to (3.5) that will in general include some logarithmic terms.

\section{Acknowledgement}

The authors are grateful for the financial support extended by SIDA project 316-2014 "Capacity building in Mathematics and its applications" under the SIDA bilateral program with Makerere University 2015-2020. The authors further extend their gratitude to the research support provided by the International Science Program (ISP). 


\section{References}

[1] L. Amphon and N. Kamsing, On the generalized solutions of a certain fourth order Euler equations, Journal of Nonlinear Sciences and Applications 10(8) (2017), 4077-4084.

DOI: http://dx.doi.org/10.22436/jnsa.010.08.04

[2] H. Azad, A. Laradji and M. T. Mustafa, Polynomial solutions of differential equations, Advances in Difference Equations, Springer Open Journal (2011), 1-12, Article 58.

DOI: https://doi.org/10.1186/1687-1847-2011-58

[3] A. E. Coddington, An Introduction to Ordinary Differential Equations, Prentice-Hall Mathematics Series, Prentice-Hall Inc., Englewood Cliffs, N. J., 1961.

[4] B. Fisher and K. Tas, On the composition of distributions $x_{+}^{-1} \ln |x|$ and $x_{+}^{r}$, Integral Transforms and Special Functions 16(7) (2005), 533-543.

DOI: https://doi.org/10.1080/10652460500105438

[5] B. Fisher, A. Kananthai, G. Sritanatana and K. Nonlaopon, The composition of the distributions $x_{-}^{-m s} \ln x_{-}$and $x_{+}^{r-\frac{p}{m}}$, Integral Transforms and Special Functions 16(1) (2005), 13-19.

DOI: https://doi.org/10.1080/10652460412331270661

[6] B. Fisher, S. Orankitjaroen, T. Kraiweeradechachai, G. Sritanratana and K. Nonlaopon, On the composition of distributions $x_{+}^{\lambda} \ln x^{m} x_{+}$and $x_{+}^{\mu}$, East-West Journal of Mathematics 9(1) (2007), 69-79.

[7] I. M. Gel'fand and G. E. Shilov, Generalized Functions, Volume 1: Properties and Operations, Translated by E. Saletan, Academic Press, New York/London, 1964.

[8] L. Hörmander, The Analysis of Linear Partial Differential Operators I, Distribution Theory and Fourier Analysis, Reprint of the Second Edition 1990, Springer-Verlag, New York, 1990.

[9] E. L. Ince, Ordinary Differential Equations, Reprinted Paper Back Edition, Dover Publications, New York, 1956.

[10] R. P. Kanwal, Generalized Functions: Theory and Technique, Mathematics in Science and Engineering, Volume 171, Academic Press, New York, 1983.

[11] L. L. Littlejohn and R. P. Kanwal, Distributional solutions of the hypergeometric differential equations, Journal of Mathematical Analysis and Applications 122(2) (1987), 325-345.

DOI: https://doi.org/10.1016/0022-247X(87)90263-0

[12] A. Kananthai, The distribution solutions of the third order Euler equation, Southeast Asia Bulletin of Mathematics 23(1999), 627-631. 
[13] A. Kananthai, The Distribution solutions to ordinary differential equations with polynomial coefficients, Southeast Asian Bulletin of Mathematics 25(1) (2001), 129-134.

DOI: https://doi.org/10.1007/s10012-001-0129-7

[14] A. Laradji, On linear equations with general polynomial solutions, Journal of Differential Equations 264(8) (2018), 5480-5488.

DOI: https://doi.org/10.1016/j.jde.2018.01.002

[15] T. Mandai, Existence of distribution null-solutions to every Fuchsian partial differential operator, Journal of Mathematical Sciences, University of Tokyo, Japan 5(1) (1998), 1-18.

[16] G. I. Mirumbe and V. A. Ssembatya, On the coexistence of distributional and rational function solutions to ordinary differential equations with polynomial coefficients, Journal of Pure and Applied Mathematics: Advances and Applications 8(2) (2012), 75-92.

[17] G. I. Mirumbe, V. A. Ssembatya, R. Bøgvad and J. E. Björk, On the distribution solutions to ordinary differential equations with polynomial coefficients on the real line, Journal of Pure and Applied Mathematics: Advances and Applications 6(2) (2011), 135-155.

[18] I. Opio, G. I. Mirumbe, J. Ssebuliba and J. M. Mango, On the solution space of ordinary differential equations with polynomial coefficients, Far East Journal of Mathematical Sciences 101(1) (2017), 103-118.

DOI: http://dx.doi.org/10.17654/MS101010103

[19] J. Wiener and K. L. Cooke, Coexistence of analytic and distributional solutions for linear differential equations, Journal of Mathematical Analysis and Applications 148(2) (1990), 390-421.

DOI: https://doi.org/10.1016/0022-247X(90)90009-5

[20] S. M. Shah and J. Wiener, Distributional and entire solutions of ordinary differential and functional differential equations, International Journal of Mathematics and Mathematical Sciences 6(2) (1983), 243-269.

DOI: http://dx.doi.org/10.1155/S0161171283000216 